\title{
Inteligencia emocional en niños de preescolar y su relación en su entorno escolar
}

\author{
Emotional intelligence in preschool children and their \\ relationship in their school environment
}

\author{
Lic. Nadia Castro Tigrero ${ }^{1}$ \\ 1986nadiacastro@gmail.com \\ Mg. Wilson Paredes Garcés ${ }^{1}$ \\ gparedes112004@hotmail.com \\ Mg. Mauricio Salas Monteros \\ ingmauriciosalas@hotmail.com
}

Recibido: 1/04/2018, Aceptado: 1/06/2018

\begin{abstract}
RESUMEN
El hombre ha sido siempre infravalorado su aspecto emocional, a tal punto de calificarlo de debilidad humana, pero en los últimos años ha surgido una corriente integradora que espera dar relevancia a este aspecto. El trabajo que realizamos fue, aplicar un instrumento de "Escala de medición Inteligencia Emocional" para niños de 5 a 7 años, basándonos en los componentes que expone Daniel Goleman, con este realizamos una prueba de consistencia y otra para medir "las habilidades de interacción social" y posteriormente lo aplicamos en 80 niños de dos Escuelas Fiscales del cantón de Milagro de la provincia del Guayas. Se utilizó el método descriptivo y no experimental para recopilar información verás por medio de la observación sistemática. Con este método se utilizó los instrumentos: como: "La subescala de medición de inteligencia emocional" y el test "Habilidades de interacción social" en la cual nos permitió medir el grado de inteligencia emocional que los niños poseen y cómo se desenvuelve en su entorno al momento de relacionarse con los demás y la forma espontánea que expresa sus emociones y más bien el control y regulación de sus emociones.
\end{abstract}

Palabras clave: Inteligencia emocional, habilidades sociales, emociones, control emocional

\footnotetext{
1 Universidad Técnica de Ambato, Ecuador
} 


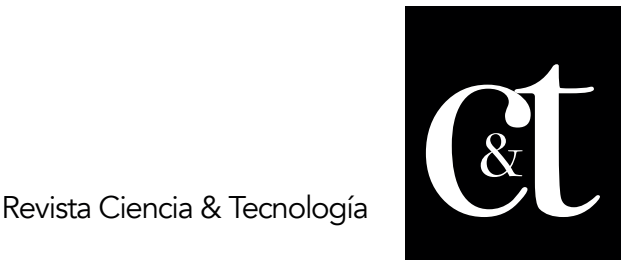

No. 19, 31 de julio de 2018

ISSN impreso: 1390 - 6321

\begin{abstract}
Man have always been undervalued their emotional aspect, to the point of describing it as human weakness, but in recent years an integrating current has emerged that hopes to give relevance to this aspect. The work we did was to apply an instrument of "Emotional Intelligence Measurement Scale" for children from 5 to 7 years old, based on the components that Daniel Goleman exhibits, with this we perform a consistency test and another to measure "interaction skills" social "and later we applied it in 80 children of two fiscal schools of the canton of Milagro of the province of Guayas. The descriptive and non-experimental method was used to collect information you will see through systematic observation. With this method the instruments were used: such as: "The emotional intelligence measurement subscale" and the "Social interaction skills" test in which it allowed us to measure the degree of emotional intelligence that children have and how it develops in their environment at the moment of relating to others and the spontaneous way that expresses their emotions and rather the control and regulation of their emotions.
\end{abstract}

Keywords: Emotional intelligence, social skills, emotions, emotional control

\title{
Introducción
}

Cuando hablamos de emociones hacemos referencia al estado de ánimo de cada persona ocasionados por diferentes factores como: los factores biológicos, que son factores genéticos y los factores psicológicos que en ella se desarrolla la depresión severa. En estos factores se demuestran los sentimientos e ideas que cada uno de ella se manifiestan a través de gestos o actitudes.

Desde la década de los noventa muchos autores han venido creando diferentes conceptos sobre la inteligencia emocional hasta la actualidad como ha sido: Daniel Goleman (1995), Fernández-Berrocal y Ruiz (2008), Mayer y Salovey (1990) y algunos en la actualidad como la Dra. Elia López (Moreno, 2015). Investigadores que han dedicado toda su vida a estudiar la inteligencia emocional y a conocer la relación que existe con otros factores, estudios que son desde las edades mayores hasta los infantes.

Haciendo referencia de los autores mencionados en el párrafo anterior podríamos decir que; la inteligencia emocional es la capacidad que tienen un individuo para recocer, comprender, regular y controlar sus emociones y así posibilitar las relaciones entre otros. Los problemas emocionales en los niños se presentan desde que nacen hasta el proceso de todo su crecimiento, a los dos años edad, estos problemas podrían variar entre los cuales tenemos: problemas de cambio de humor; problemas de violencia; problemas de autoestima, entre otros. Por aquello los niños demuestran diferentes formas de manifestar sus emociones haciendo un sin números de rabietas y berrinches, llantos incontrolables y que, en algunos de los casos, los padres podrían llegar a pensar que son comportamientos normales para su corta edad. Por tal situación los padres deben inculcar el comportamiento a temprana edad ya que la primera escuela de los niños es la familia, desde luego es de suma importancia que los padres instauren un estado emocional en los niños desde que nacen hasta la edad preescolar, por tanto, esto logrará alcanzar el control de sus emociones. Asumiendo que este hecho no se puede hacer solo con decirlo, sino más bien enseñándole con la réplica de sus acciones, para que así los 


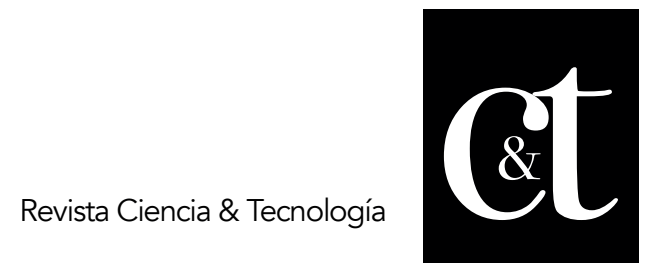

No. 19, 31 de julio de 2018

ISSN impreso: 1390 - 6321

niños pongan en práctica, el saber controlar y regular sus emociones.

Al estudiar la inteligencia emocional nos direccionamos a las escalas que me ayudan a determinar, cuáles son los indicadores que contribuyen a tener un control emocional adecuado, para poder tener la habilidad de relacionarse con las demás personas (Goleman, 1995), como es la autoconciencia, que es aquella que nos ayuda a sintonizar la información de los sentimientos, sensaciones, intenciones y acciones para comprender la forma como reaccionan las personas. Luego tenemos al autocontrol: que es la habilidad de controlar las emociones, comportamientos e impulsos, de manera que piense anticipadamente ante cualquier situación y poder tener control de sus actos. Otra de las escalas es el aprovechamiento emocional: que es la capacidad de buscar sus objetivos propios y la realización de sus metas. La empatía es habilidad de comprender a los demás y de ponerse en el lugar del otro. Y por último tenemos a las habilidades sociales: que no es más que; la capacidad de emprender buenas relaciones con los demás. La autorregulación es uno de los indicadores importantes que se inmersa en el desarrollo de la inteligencia emocional en los niños al cumplir los tres años de edad. Este indicador hace que el infante logre el control y perfeccionamiento de sí mismo, no dejando a un lado al adulto, no deja de cumplir su papel relevante en la aportación de información sobre lo que le es o no socialmente aceptable para él niño. Es por eso que se han dado muchos estudios para poder estar al tanto de cómo se produce el reconocimiento de las emociones, y cuando se alcanza la capacidad de expresarlas y de regularlas de forma exitosa.

Esta investigación ofrece una exploración minuciosa de la inteligencia emocional en edades tempranas, ya que hemos podido notar que el inicio de las emociones se produce desde su nacimiento y es ahí; el aporte fundamental de los padres de familia y educadores, de ayudar a educar las emociones de sus pequeños, para que sean un ente productivo en conocimiento y en relaciones sociales dentro y fuera de su ambiente escolar. Por ello el objetivo de esta investigación es conocer la importancia que tiene la inteligencia emocional en los niños de preescolar, y la habilidad de relacionarse en su ambiente escolar.

Para aquello hemos aplicado un test de "Escala de dimensión de Inteligencia emocional para niños de 5 a 7 años", que nos ayudará a medir las dimensiones de la IE, y cuál de los indicadores tienen más influencia para lograr un estado emocional estable para cada uno de los niños. Para poder obtener mejores resultados también se aplicó el instrumento de "Habilidades de Interacción social" este instrumento me ayuda a conocer si los niños tienen la capacidad para comprender la conducta, defender y expresar sus inquietudes. También nos muestra si es agradable, si demuestra significativamente cariño a los demás y si tiene la capacidad de reconocer sus emociones propias y de los demás. Y por último me comprueba la capacidad que tiene para trabajar en equipo, dialogar, el grado de obediencia y el fortalecimiento que hay entre compañeros.

\section{Desarrollo}

Para conocer las emociones, es necesario tener el conocimiento de las teorías que lo fundamentan muchos autores sobre el estudio de ellas. Teorías y enfoques de las emociones El enfoque psicológico se refiere a los cambios físicos que presenta el ser humano, condicionando las emociones de los seres humanos. Esta teoría no tiene 


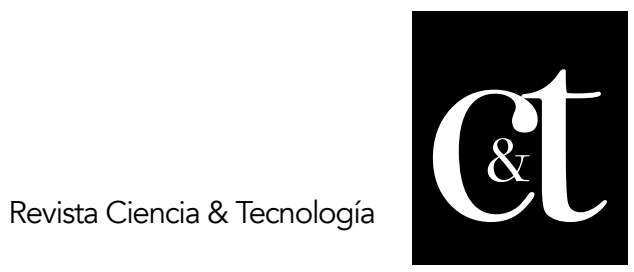

No. 19, 31 de julio de 2018

ISSN impreso: 1390 - 6321

mucha relevancia porque no realiza un acercamiento conceptual de la presencia de las emociones (Guamán Córdova, 2017).

También tenemos otro aporte sobre la distinción de la causa y el objeto de la emoción por James Lange en 1884 que nos dice: "Mientras que la primera es la idea que la suscita, la otra es la idea a la que se refiere" (Guamán Córdova, 2017), por lo tanto, esto quiere decir que la situación influye para el que es sujeto presente sus emociones y es aquí donde pone en juego el pasado de su memoria. Enfoque de la teoría evolutiva

En este enfoque tomamos hacemos referencia a la teoría de la evolución de Charles Darwin donde nos da como dice: "la expresión de las emociones en los animales y el hombre a fin de conocer la adaptación del sujeto con diferentes ambientes". Esto quiere decir que demuestra su comportamiento según el entorno donde se desenvuelven.

Teorías cognitivas que la expone Magda Arnold en su libro Emoción y personalidad (1960); Guamán Córdova (2017), nos habla sobre la cognición que esto quiere decir: la evaluación que hace que el sujeto en bien o en mal de alguna situación, ya que aquí aparecen la emociones. Daniel Goleman (1995) nos aporta concepción de la inteligencia emocional que nos dice: "conocer las propias emociones; manejar las emociones, motivarse a sí mismo; reconocer las emociones de los demás y establecer relaciones" (Pina, 2017), también nos habla sobre: "conciencia emocional; regulación emocional; autonomía personal; inteligencia interpersonal y habilidades para la vida y bienestar".

Bisquerra (2003), en un trabajo de análisis de fundamentación de educación emocional, nos indica: "Que el conjunto de competencias emocionales que le sirve de base" (Pina, 2017), por tanto, el estudio de cada uno de estos autores ratifica que, al reconocer, manejar, regular, y motivarse así mismos en base a sus emociones contribuirán a que los niños puedan relacionarse de mejor manera en su entorno.

Piaget nos dice "las emociones cambian a medida que se van desarrollando nuevas habilidades para interpretar las relaciones sociales". Kohlberg (1964) reconoce "la importancia de las emociones tanto en el espacio social y en el aspecto moral" (Camacho, Ordoñez, Roncancio, y Vaca, 2017) esto quiere decir que es donde el individuo hace transgresión con vergüenza, culpabilidad o miedo, donde se realiza una serie de diferencias cognitivas en relación al razonamiento moral del sujeto. También algunos autores como Peter Salovey y John Mayer que en 1990 crean unos programas de SEL, debido a que había la necesidad de formar habilidades básicas direccionadas con la IE, como la percepción emocional, regulación, comprensión, personalidad, control, tales como la autoestima y el asertividad (Fernandez- Berrocal y Ruiz, 2008).

Nos podemos dar cuenta que cada uno de los indicadores que hemos fundamentado tienen efectos positivos en el ambiente familiar y escolar para crear unos vínculos de armonía y relajación entre los estudiantes y su entorno que lo rodea (Manríquez, 2014). 


\section{Metodología}

El diseño de esta investigación se efectuará a través de los siguientes tipos de estudio: Consideramos que es aplicada, porque en el proceso de nuestro trabajo investigativo, contamos con la colaboración de los directivos de la E.E.B. "Isabel Herrera de Velázquez" y E.E.B "Victoria Macías de Acuña".

También este estudio lo consideramos teórico porque está fundamentada con la bibliografía, que se ha recopilado en el transcurso de la revisión de la literatura, para así tener las bases sólidas de lo que es la inteligencia emocional y de qué manera influyen en la habilidad para relacionarse en su ambiente escolar.

Mediante esta investigación se conocerá las características descriptivas de la población de una manera exacta, que ayudará a identificar cada una de las variables de estudios. Poniendo en conocimiento que nuestro objetivo es medir el estado emocional de los infantes y a su vez observar de forma directa, la habilidad que tiene para relacionarse y expresar sus emociones en determinadas situaciones.

Para la elaboración de este trabajo se escogió a una población de niños y niñas entre las edades de 5 a 6 años, de los niveles de inicial II y primero de Básica. El total de los niños evaluados son de 80 niños de 5 años de edad. Que esto comprende en 25 niños y 15 niñas que hacen un total de 40 niños de la E.E.B "Isabel Herrera de Velázquez" y 18 niños y 22 niñas que hacen un total de 40 niños de la E.E.B "Victoria Macías de Acuña". Estos infantes fueron escogidos para poder aplicar los instrumentos como: "La subescala de medición de inteligencia emocional" y el test "Habilidades de interacción social".

"La subescala de medición de inteligencia emocional" (Psicología, 2004) es un instrumento de medición, consta con 5 subescalas y tiene 15 ítems. Las subescalas que divide la prueba son: autoconciencia, autocontrol, aprovechamiento emocional, empatía y habilidades sociales. Y también consta de 15 láminas para relacionar con cada uno de los ítems, las láminas constan con dibujos que ayudaran al participante a entender de manera clara la situación que se está preguntando.

Para la toma de este instrumento será de 10 a 15 minutos por infante. Cada ítem de las subescalas tenía 3 a 5 opciones de respuesta y se le dio la calificación (1); (2) y (3) para así tener un puntaje.

Test de "Habilidades de interacción social" está estructurado por tres dimensiones como son: la autoafirmación, la expresión de las emociones y habilidades para relacionarse, ya que al medir podemos lograr un alto nivel de validez del instrumento.

La dimensión "Autoafirmación" consta de 10 ítems, la dimensión "Expresión de emociones" cuenta con 8 ítems y por último la "Habilidades para relacionarse" cuenta con 6 ítems y los puntajes se obtienen a través de una escala de Likert (nunca, pocas veces, algunas veces, muchas veces y siempre) siendo 1 el valor mínimo a seleccionar "nunca" y el 5 máximo a seleccionar "siempre". Se cuenta con la tabla de los baremos elaborados en base a puntuaciones transformadas a percentiles, para cada una de las dimensiones y relacionadas a el valor total de la 


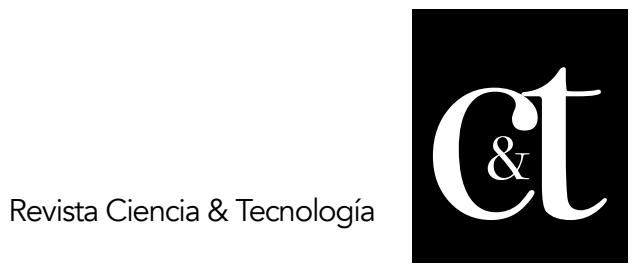

No. 19, 31 de julio de 2018

ISSN impreso: 1390 - 6321

prueba. El valor máximo de la prueba es de 120 y el mínimo de 42 considerando que pocas veces seria nuestro valor mínimo, en la dimensión de "Autoafirmación" el valor del puntaje estaría de 50 como máximo y 10 como mínimo. Con la dimensión de "Expresión de las emociones" su puntaje es de 40 como máximo y 12 como mínimo y, por último, para la dimensión "Habilidades para relacionarse" el puntaje máximo es de 30 y un mínimo de 11.

\section{Procedimiento}

Lo primero que se realizó es pedir la debida autorización a los directivos de las instituciones como de la Escuela de Educación Básica "Isabel Herrera de Velásquez" y E.E.B "Victoria Macías de Acuña", para que nos autorice aplicar los test a los niños de nivel de inicial II y de primero de básica.

Para la aplicación del instrumento "La subescala de medición de inteligencia emocional" se escogió a los participantes de forma individual y al azar pero que estén dentro del rango de edad, al evaluar a cada niño se le explicó como era el proceso, en las cuales se le exponía la lámina e inmediatamente se le ejecutaba las preguntas, y se les indicaba las opciones que tiene cada pregunta. Y así obtener las respuestas claras y sinceras. También se aplicó el test. "Habilidades de Interacción social" en las cuales se le realizaba un cuestionario de preguntas.

Análisis de Datos Para el respectivo análisis de datos se utilizó el programa SPSS v 25.0 realizando los cálculos estadísticos descriptivos. Este trabajo tiene como objetivo realizar la descripción de la inteligencia emocional y cual son los indicadores que ayudan a obtener el estado emocional adecuado para poder adquirir la habilidad para relacionarse entre los participantes y quienes forman parte de su ambiente escolar.

\section{Resultados}

Con el fin de profundizar el estudio, se aplica análisis univariante para relacionar cada dimensión (subescalas) de la variable independiente, con sus indicadores respectivos; con el percentil de la prueba total, obtenidos de los test de "escala de medición de inteligencia emocional" (variable dependiente) y de "Habilidades de interacción social".

La inferencia estadística, por subescala, se muestra a continuación: 


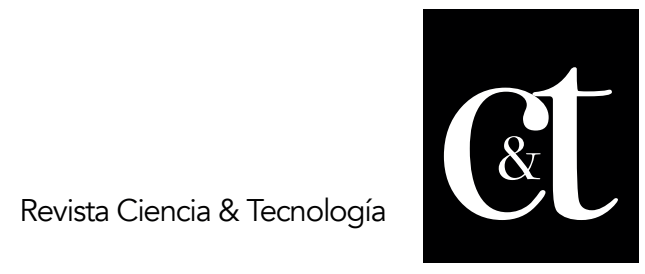

No. 19, 31 de julio de 2018

ISSN impreso: 1390 - 6321

\section{Autoconciencia}

Tabla 1. Pruebas de los efectos inter-sujetos variable Autoconciencia

\begin{tabular}{|c|c|c|c|c|c|}
\hline Origen & $\begin{array}{c}\text { Suma de } \\
\text { cuadrados } \\
\text { tipo III } \\
\end{array}$ & gl & $\begin{array}{c}\text { Media } \\
\text { cuadrática }\end{array}$ & $\mathrm{F}$ & Sig. \\
\hline $\begin{array}{l}\text { Modelo corregido } \\
\text { Intersección } \\
\text { Reacción }\end{array}$ & $\begin{array}{r}7173,887^{a} \\
163775,896 \\
1050,604\end{array}$ & $\begin{array}{r}32 \\
1 \\
3\end{array}$ & $\begin{array}{r}224,184 \\
163775,896 \\
350,201\end{array}$ & $\begin{array}{r}, 961 \\
702,099 \\
1,501\end{array}$ & $\begin{array}{l}, 540 \\
, 000 \\
, 226\end{array}$ \\
\hline Comprensión & 576,249 & 2 & 288,125 & 1,235 & ,300 \\
\hline Conciencia & 553,605 & 3 & 184,535 & ,791 & ,505 \\
\hline Reacción * Comprensión & 1605,657 & 5 & 321,131 & 1,377 &, 250 \\
\hline Reacción * conciencia & 594,526 & 7 & 84,932 & , 364 & ,918 \\
\hline $\begin{array}{l}\text { Comprensión * } \\
\text { conciencia }\end{array}$ & 2852,616 & 6 & 475,436 & 2,038 & ,079 \\
\hline $\begin{array}{l}\text { Reacción * Comprensión } \\
\text { * conciencia }\end{array}$ & 937,444 & 6 & 156,241 & 670 & ,674 \\
\hline Error & 10963,500 & 47 & 233,266 & & \\
\hline Total & 439653,000 & 80 & & & \\
\hline Total corregida & 18137,387 & 79 & & & \\
\hline
\end{tabular}

a. $R$ cuadrado $=, 396(R$ cuadrado corregida $=-, 016)$

Los resultados del análisis univariante muestran que ningún indicador de la autoconciencia influye en las habilidades para relacionarse en su ambiente escolar.

\section{Autocontrol}

Tabla 2. Pruebas de los efectos inter-sujetos variable Autocontrol

Pruebas de los efectos inter-sujetos

\begin{tabular}{|l|r|r|r|r|r|}
\hline Variable dependiente: Percentil & $\begin{array}{c}\text { Suma de } \\
\text { cuadrados tipo } \\
\text { Origen }\end{array}$ & GI & \multicolumn{1}{c|}{$\begin{array}{c}\text { Media } \\
\text { cuadrática }\end{array}$} & \multicolumn{1}{c|}{ F } & Sig. \\
\hline Modelo corregido & $10154,340^{\mathrm{a}}$ & 31 & 327,559 & 1,970 &, 017 \\
Intersección & 121345,763 & 1 & 121345,763 & 729,621 &, 000 \\
Decisión & 802,076 & 3 & 267,359 & 1,608 &, 200 \\
Control & 1813,217 & 4 & 453,304 & 2,726 &, 040 \\
Regulación & 984,882 & 2 & 492,441 & 2,961 &, 061 \\
Decisión * Control & 1334,313 & 6 & 222,386 & 1,337 &, 259 \\
Decisión * Regulación & 1131,544 & 5 & 226,309 & 1,361 &, 256 \\
Control * Regulación & 2692,339 & 6 & 448,723 & 2,698 &, 024 \\
Decisión * Control * & 813,879 & 4 & 203,470 & 1,223 &, 313 \\
Regulación & 7983,048 & 48 & 166,313 & & \\
Error & 439653,000 & 80 & & & \\
Total & 18137,387 & 79 & & & \\
Total corregida & & & & & \\
\hline
\end{tabular}

a. $\mathrm{R}$ cuadrado $=, 560(\mathrm{R}$ cuadrado corregida $=, 276)$ 
Los resultados del análisis univariante muestran que el autocontrol influye en las habilidades para relacionarse en su ambiente escolar, cuando el control de las emociones actúa de manera individual o asociado con la regulación de las emociones.

Considerando que el indicador "control", si influye para tener una inteligencia emocional, ya que el niño tendrá el dominio de controlar sus emociones, sentimientos, impulsos y comportamientos, de esa manera podrá obtener una excelente relación con los demás. Y si se fusiona el control y la regulación que no es más que disminuir la intensidad de sus emociones sean estas positivas o negativas alcanzara las habilidades para relacionarse.

\section{Aprovechamiento emocional}

Tabla 3. Pruebas de los efectos inter-sujetos variable Aprovechamiento emocional

Variable dependiente: Percentil

\begin{tabular}{|c|c|c|c|c|c|}
\hline Origen & $\begin{array}{c}\text { Suma de } \\
\text { cuadrados tipo } \\
\text { III }\end{array}$ & $\mathrm{Gl}$ & $\begin{array}{c}\text { Media } \\
\text { cuadrática }\end{array}$ & $\mathrm{F}$ & Sig. \\
\hline Modelo corregido & $6727,499^{a}$ & 16 & 420,469 & 2,322 & ,009 \\
\hline Intersección & 66402,268 & 1 & 66402,268 & 366,642 &, 000 \\
\hline Expresión & 249,053 & 2 & 124,526 & ,688 & ,507 \\
\hline Responsabilidad & 903,502 & 3 & 301,167 & 1,663 & ,184 \\
\hline Asertividad & 2652,013 & 3 & 884,004 & 4,881 & ,004 \\
\hline Expresión * & & & & & \\
\hline Responsabilidad & 997,856 & 3 & 332,619 & 1,837 & 150 \\
\hline Expresión * Asertividad & 907,367 & 2 & 453,683 & 2,505 & ,090 \\
\hline Responsabilidad * & 1270567 & 3 & 156856 & 2522 & $0 G 6$ \\
\hline Asertividad & | & 3 & 400,050 & $2,0<3$ & لي ب06 \\
\hline Expresión * & & & & & \\
\hline Responsabilidad * &, 000 & 0 & & & \\
\hline Asertividad & & & & & \\
\hline Error & 11409,889 & 63 & 181,109 & & \\
\hline Total & 439653,000 & 80 & & & \\
\hline Total corregida & 18137,387 & 79 & & & \\
\hline
\end{tabular}

a. R cuadrado $=, 371$ ( $R$ cuadrado corregida $=, 211)$ 
Los resultados del análisis univariante muestran que el aprovechamiento emocional influye en las habilidades para relacionarse en su ambiente escolar, cuando el asertividad actúa de manera individual.

Considerando que el asertividad es importante en la inteligencia emocional ya que contribuye a que los niños puedan comprender su forma de actuar y que logren comunicarse con los demás, defendiendo sus opiniones y derechos sin afectar o pisotear a los otros. Además, podrán exponer críticas constructivas y logra reconocer los errores.

\section{Empatía}

\section{Tabla 4. Pruebas de los efectos inter-sujetos variable Empatía}

Varlable dependiente: Percentll

\begin{tabular}{|c|c|c|c|c|c|}
\hline Origen & $\begin{array}{c}\text { Suma de } \\
\text { cuadrados tipo III }\end{array}$ & gl & Media cuadratica & $\mathrm{F}$ & Sig. \\
\hline Modelo corregido & $4997,792^{\mathrm{a}}$ & 27 & 185,103 &, 733 & 808 \\
\hline Intersección & 114953,687 & 1 & 114953,687 & 454,930 & .000 \\
\hline Interpersonal & 126,718 & 2 & 63,359 & .251 &., 779 \\
\hline Empáticos & 44,709 & 2 & 22,355 & ,088 & ;915 \\
\hline Solidaridad & 444,039 & 4 & 111,010 & ,439 &, 780 \\
\hline Interpersonal "Empäticos & 659,196 & 2 & 329,598 & 1,304 &, 280 \\
\hline Interpersonal 'Solidaridad & 658,577 & 5 & 131,715 & 521 &, 759 \\
\hline Empáticos ' Solldaridad & 1328,411 & 8 & 166,051 & ,657 &, 726 \\
\hline Interpersonal 'Empáticos' & 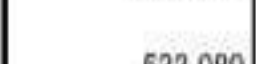 & & 000 & & \\
\hline Solidaridad & 003,004 & ग & 17,696 &, 003 & 554 \\
\hline Error & 13139,595 & 52 & 252,685 & & \\
\hline Total & 439653,000 & 80 & & & \\
\hline Total corregida & 18137,387 & 79 & & & \\
\hline
\end{tabular}

Fuente tabulada del programa SPSS 20.0

a. $\mathrm{R}$ cuadrado $=, 276$ ( $\mathrm{R}$ cuadrado corregida $=-, 101)$

Los resultados del análisis univariante muestran que ningún indicador de la empatía influye en las habilidades para relacionarse en su ambiente escolar. 


\section{Habilidades Sociales}

\section{Tabla 5. Pruebas de los efectos inter-sujetos variable Habilidades sociales}

\begin{tabular}{|c|c|c|c|c|c|}
\hline Origen & $\begin{array}{c}\text { Suma de } \\
\text { cuadrados tipo III }\end{array}$ & gl & Media cuadratica & $\mathrm{F}$ & Sig: \\
\hline Modelo corregido & $3273,175^{\mathrm{a}}$ & 20 & 163,659 &, 650 &, 857 \\
\hline Intersección & 89615,753 & 1 & 89615,753 & 355,709 &, 000 \\
\hline Relacionarse & 551,169 & 2 & 275,584 & 1,094 &, 342 \\
\hline Interacción & 158,365 & 2 & 79.182 & .314 & .732 \\
\hline Defensa & 68,928 & 3 & 22,976 &, 091 & 965 \\
\hline Relacionarse " Imteracción & 219,541 & 2 & 109,771 &, 436 &, 649 \\
\hline Relacionarse ${ }^{\circ}$ Defensa & 150,382 & 3 & 50,127 & .199 &, 897 \\
\hline Interacción ' Defensa & 313,905 & 5 & 62,781 & 249 & ,939 \\
\hline Relacionarse * Interacción * & & & & & \\
\hline Defensa & 28,516 & 2 & 14,258 &, 057 & ,945 \\
\hline Error & 14864,212 & 59 & 251,936 & & \\
\hline Total & 439653,000 & 80 & & & \\
\hline Total corregida & 18137,387 & 79 & & & \\
\hline
\end{tabular}

Fuente tabulada del programa SPSS 20.0

a. R cuadrado $=, 180$ ( $R$ cuadrado corregida $=-, 097$ )

Los resultados del análisis univariante muestran que ningún indicador de la habilidad social influye en las habilidades para relacionarse en su ambiente escolar.

\section{Tabla 6. Estadísticos}

\begin{tabular}{|c|c|c|c|c|c|c|}
\hline \multicolumn{7}{|c|}{ Estadisticos } \\
\hline & & Rel & Auto & Expresion & Total & Percentil \\
\hline \multirow{2}{*}{ N } & Validos & 80 & 80 & 80 & 80 & 80 \\
\hline & Perdidos & 0 & 0 & 0 & 0 & 0 \\
\hline \multicolumn{2}{|c|}{ Media } & 40,4125 & 33,3250 & 26,0250 & 99,7125 & 72,5875 \\
\hline \multicolumn{2}{|c|}{ Mediana } & 41,0000 & 34,0000 & 27,0000 & 99,0000 & 75,0000 \\
\hline \multicolumn{2}{|c|}{ Moda } & 41,00 & 34,00 & 27,00 & 99,00 & 75,00 \\
\hline \multicolumn{2}{|c|}{ Desv, tip. } & 5,04836 & 4,19697 & 3,24125 & 8,17405 & 15,15213 \\
\hline \multicolumn{2}{|c|}{ Varianza } & 25,486 & 17,615 & 10,506 & 66,815 & 229,587 \\
\hline \multicolumn{2}{|c|}{ Asimetria } &,- 371 & 318 &,- 574 &, 057 &,- 960 \\
\hline \multicolumn{2}{|c|}{ Error tip. de asimetria } & .269 & 269 & .269 & .269 & 269 \\
\hline \multicolumn{2}{|c|}{ Curtosis } & .350 & .515 & 1,139 & .784 & 1,895 \\
\hline \multicolumn{2}{|c|}{ Error tip. de curtosis } & .532 &, 532 &, 532 & .532 &, 532 \\
\hline
\end{tabular}

Fuente tabulada del programa SPSS 20.0 
Los resultados indican que tenemos una Media $=(72,58)$, una Desv. Tip. $=(15,15)$ esto nos muestra que los valores están entre $+0-$, esto quiere decir que la tendencia están entre 67 y 77, de la misma manera tenemos una varianza ( $229,58)$, y una Asimetría $=(-0,96)$ quiere decir que las mayoría de los evaluados califica alto, ya que la asimetría tiene una desviación a la izquierda, también tenemos una Curtosis positiva $(1,89)$ esto quiere decir que nuestro datos están alrededor de mi media aritmética. También tenemos una Mediana $=75$ que esto señala en términos generales que tenemos un Percentil de 75 lo que nos indica que los estudiantes de las instituciones, están en las condiciones de poder relacionarse en su ambiente escolar, pero siempre y cuando trabajando los indicadores de la inteligencia emocional: el control, regulación y asertividad, ya que estos factores pueden establecer conexión favorable para relacionarse de mejor manera.

\section{Conclusiones}

Enmarcándonos en los resultados obtenidos del estudio de la inteligencia emocional, comprobamos que los indicadores que forma la Inteligencia Emocional en los niños son el control, la regulación y asertividad. Por tal comprobación podemos decir que la inteligencia emocional, es de suma importancia desarrollarla para que el individuo, en este caso el niño, se relacione con su entorno escolar, de una manera accesible y empática, dado que estos indicadores cumplen su papel importante en los infantes ya que se demostró que los niños pueden relacionarse con las personas de su entorno escolar, aunque su inteligencia emocional sea baja no influye, pero si se desarrolla una excelente inteligencia emocional, el niño podrá adquirir la habilidad de relacionarse de formar permanente y armónica entre los demás.

Es por eso que resultados obtenidos en esta investigación, muestran que después de haberse aplicado los test de inteligencia emocional, con estudiantes de 5 años, efectivamente la inteligencia emocional juega un papel muy importante para desarrollar la habilidad social en los niños. Por lo anterior, se acepta la hipótesis planteada: que, desarrollando una inteligencia emocional, los niños mejoran las habilidades sociales. Por otro lado, se comprueba que los test aplicados fueron significativos, logrando detectar si los niños muestran control de sus emociones, regulación de sus emociones y autoconfianza, para poder ver si los niños muestran habilidad social. Afianzando lo expuesto por (Goleman, 1995). También se fundamenta con lo expuesto en la teoría de James Lange de 1884 y posteriormente la teoría de Cannon-Bard (Fernández et al., 2010).

En definitiva, se recomienda que para estudios posteriores se pueda hacer el mismo diseño de proyecto, para poder obtener un instrumento para valorar objetivamente la inteligencia emocional de los niños y utilizarlo para la atención médica familiar.

\section{Referencias bibliográficas}

Arnold, Magda (1960). Emotions and personality. New York: Columbia University Press.

Bisquerra Alzina, R. (2003). Educación emocional y competencias básicas para la vida. Revista De Investigación Educativa, 21(1), 7-43. Recuperado a partir de 
https://revistas.um.es/rie/article/view/99071

Camacho, B. N. M., Ordoñez, L. J. C., Roncancio, A. M. H., y Vaca, V. P. (2017). Convivencia escolar y cotidianidad: una mirada desde la inteligencia emocional. Revista Educación y Desarrollo Social, 11(1), 2011-5318. https://doi.org/10.18359/reds.2649

Fernández, E.G.; García, B.; Jiménez, M.P.; Martín, M.D. y Domínguez, F.J. (2010). Psicología de la emoción. Editorial Universitaria Ramón Areces: Madrid.

Fernández-Berrocal, P. \& Ruiz, D. (2008). Emotional intelligence in education. The Electronic Journal of Research in Educational Psychology, 15(6), 421- 436.

Goleman, D. (1995). La inteligencia emocional. Bantam Books.

Guamán Córdova, W. F. (2017). Propuesta para la implementación del rincón de las emociones en primer año de educación general básica. Retrieved from http://dspace.ucuenca.edu.ec/handle/123456789/28399

Kohlberg, L. (1964). Development of moral character and moral ideology. In M.L. Hoffman, \& L.W. Hoffman (Eds.), Review of child development research (Vol. 1, 381-431). New York, USA: Russel Sage Foundation.

Manríquez, M. S. (2014). Convivencia Y Clima Escolar: Claves De La Gestión del Conocimiento. Última Década, (núm. 41), 153-178. Retrieved from http://www.redalyc.org/articulo.oa?id=19536988007 Méndez Calle, M. J., \&

Mayer, J.D. \& Salovey, P. (1990). What is emotional intelligence? In P. Salovey \& D. Sluyter (Eds). Emotional Development and Emotional Intelligence: Implications for Educators (p. 3-31) Nueva York: Basic Books.

Moreno, J. M. (2015). Inteligencia emocional en la etapa de Educación Infantilde las emociones primarias a las secundarias. Trabajo de fin de grado, Universidad de Granada.

Pina, M. B. (2017). Diversidad educativa ¿Un potencial desconocido? Revista de Investigación Educativa, https://doi.org/10.6018/rie.35.1.275031 